\title{
Outcomes of endovascular interventional therapy for primary Budd-Chiari syndrome caused by hepatic venous obstruction
}

\author{
DE-LEI CHENG ${ }^{1,2^{*}}$, NAN ZHU ${ }^{3 *}$, HAO XU ${ }^{4}$, CHENG-LI LI $^{1}$, WEI-FU LV ${ }^{2}$, \\ WEI-WEI FANG ${ }^{2}$ and CHUAN-TING LI ${ }^{1}$
}

\author{
${ }^{1}$ Shandong Medical Imaging Research Institute, Shandong University, Jinan, Shandong 250021; \\ ${ }^{2}$ Department of Interventional Radiology, Anhui Provincial Hospital, Hefei, Anhui 230000; ${ }^{3}$ Department of Radiology, \\ People's Hospital of Wuhe County, Bengbu, Anhui 233300; ${ }^{4}$ Department of Interventional Radiology, \\ Affiliated Hospital of Xuzhou Medical University, Xuzhou, Jiangsu 221100, P.R. China
}

Received March 11, 2018; Accepted August 9, 2018

DOI: $10.3892 /$ etm.2018.6708

\begin{abstract}
To date, interventional therapy for patients with Budd-Chiari syndrome (BCS) due to hepatic vein obstruction (HVO) has not been standardized in China. In Western countries, BCS primarily occurs due to thrombosis and the majority of patients receive thrombolysis. In China, BCS is mostly caused by the membranous occlusion of the HV or IVC. The present retrospective study evaluated the efficacy of recanalization techniques in patients with primary BCS due to HVO. The data of 69 patients with BCS due to HVO, who underwent endovascular therapy at 2 centers in China between December 2010 and December 2012, were analyzed. All of the patients underwent balloon angioplasty. In addition, 14, 6 and 5 patients received thrombolysis, endovascular stent and thrombolysis + endovascular stent, respectively. The overall technical success rate was $95.7 \%$ (66/69), and was comparable among the treatments. The HV pressure after the treatments was significantly lower compared with that prior to the procedures $(23.3 \pm 6.9$ vs. $\left.46.5 \pm 8.6 \mathrm{cmH}_{2} \mathrm{O} ; \mathrm{P}<0.001\right)$. The mean follow-up duration was 75 months (range, 60-84 months). During the 5-year follow-up, 10 patients (15.2\%) had developed a recurrence of BCS-associated symptoms, of which 7 were successfully treated. The cumulative survival rates at 12, 36 and 60 months after endovascular interventional therapy (balloon angioplasty or combined treatment) were 98.5,98.5 and 93.9\%, respectively. After treatment by endovascular therapy, the patients with BCS caused by HVO had high survival rates and low recurrence rates in the short- and mid-term.
\end{abstract}

Correspondence to: Professor Chuan-Ting Li, Shandong Medical Imaging Research Institute, Shandong University, 32 Jing Five Road, Jinan, Shandong 250021, P.R. China

E-mail: lichuanting123@yeah.net

${ }^{*}$ Contributed equally

Key words: Budd-Chiari syndrome, hepatic vein, interventional therapy, angioplasty, thrombolysis, stenting

\section{Introduction}

Budd-Chiari syndrome (BCS) is a clinical disorder caused by the obstruction of the hepatic vein (HV) outflow system, anywhere from the hepatic venules to the cavoatrial junction (1-3). BCS may be caused by obstruction of the HV and/or inferior vena cava (IVC) $(1,3,4)$. Patients with BCS suffer from liver injury secondary to the obstruction of venous outflow, leading to progressive symptoms and even liver cirrhosis if not treated in a timely manner. Patients with BCS in the acute phase may succumb to hepatic failure. In chronic BCS, patients develop hepatocirrhosis leading to various complications, including gastrointestinal bleeding, refractory ascites and hepatocellular cancer $(1,3,5)$.

The treatment of BCS has evolved considerably over the past few decades, and the overall 5-year survival rate has risen to $80-90 \%$ (4,6-8). Various treatment options for BCS include the following: i) Anti-coagulant therapy; ii) endovascular decompression therapy, including thrombolysis, stent-graft placement, angioplasty and transjugular intrahepatic portosystemic shunt (TIPS); and iii) orthotopic liver transplantation. Due to differences in the etiopathogenesis of BCS between China and western countries, treatment options vary widely. In western countries, BCS mostly occurs due to thrombosis and most patients receive thrombolysis, TIPS or liver transplantation $(9,10)$. However, in China, BCS is mostly caused by membranous occlusion of the HV or IVC. Therefore, most patients with BCS in China undergo angioplasty (11-13).

Formerly, BCS in China was thought to be due to IVC obstruction only. However, recent Chinese studies have indicated that most patients with BCS either present with an obstruction of the $\mathrm{HV}$ alone, or of the IVC and $\mathrm{HV}$ combined $(11,14)$. Therefore, in China, the endovascular treatment of $\mathrm{HV}$ obstruction (HVO) associated with BCS has become a new challenge in clinical practice.

While endovascular interventional therapy for patients with BCS due to IVC obstruction has been standardized $(15,16)$, it is still evolving for patients with BCS due to HVO. Western countries advocate the use of TIPS as a primary treatment for HVO in BCS $(3,9,10)$, while Chinese physicians prefer recanalization $(4,13,17)$. The present retrospective study assessed the 
efficacy of recanalization in 69 consecutive patients with BCS due to HVO.

\section{Materials and methods}

Patient data. All procedures were performed in accordance with the ethical standards of the responsible committee on human experimentation (institutional and national) and with the Helsinki Declaration of 1975, as revised in 2008 (5). All patients provided written informed consent prior to undergoing the procedure.

The prospectively maintained data of patients diagnosed with BCS and treated at Anhui Provincial Hospital (Hefei, China) or Affiliated Hospital of Xuzhou Medical University (Xuzhou, China) and December 2010 and December 2012 were reviewed. For inclusion in the present study, patients were required to conform to the following: Primary BCS due to HV stenosis or HV occlusion as confirmed by magnetic resonance imaging (Fig. 1) or digital subtraction angiography, and symptoms associated with portal hypertension. Patients with any of the following were excluded: Hepatic sinusoidal obstruction syndrome; IVC thrombosis; recurrent BCS; or BCS due to any other cause, including cancer, cysts or parasites. Patients who were lost to follow-up within 5 years were also excluded (Fig. 2).

Recanalization of the $H V$ via transjugular and/or femoral vein approach. The venography of the IVC was performed first, via the internal jugular and/or femoral vein, in order to identify the ostium of the $\mathrm{HV}$ and correlate it with the pre-operative images. In patients with $\mathrm{HV}$ stenosis, a 5F Cobra catheter and an ultra-slip wire were used to explore the ostium of the target HV. For patients with complete HVO (left, middle or right), a single-bend catheter and a self-made single-bend needle (Fig. 3A and B) were used to puncture the major HV (left, middle or right that was most affected by the obstruction) trunk, branch and/or traffic branch (i.e., the connecting vessel between two major HVs). After successful cannulation, HV angiography was performed, and the $\mathrm{HV}$ pressure was measured in order to assess luminal obstruction of the affected major $\mathrm{HV}$ and its branches. After complete assessment, balloon angioplasty (Figs. 4-6) and endovascular stenting were performed. The patients with BCS who underwent balloon angioplasty only, were included in balloon angioplasty group. The patients who received balloon angioplasty in addition to other methods of angioplasty, including thrombolysis and/orendovascular stenting, were included in the combination therapy group.

Recanalization of HV via percutaneous transhepatic and IVC approach. After successful percutaneous puncture of one of the branches of the HV, the guide wire was passed through the obstructed segment of the HV and then drawn out from the internal jugular or femoral vein. Subsequently, recanalization of the HV was performed in the opposite direction through the IVC using an internal jugular or femoral vein approach.

Recanalization of $H V$ with thrombus. A $5 \mathrm{~F}$ thrombolytic catheter was placed in the HV via a transjugular approach and thrombolysis was performed with urokinase $(100,000 \mathrm{U}$; 4-6 times daily). Radiography was performed every 3 days for review, and the catheter was adjusted so that the catheter's

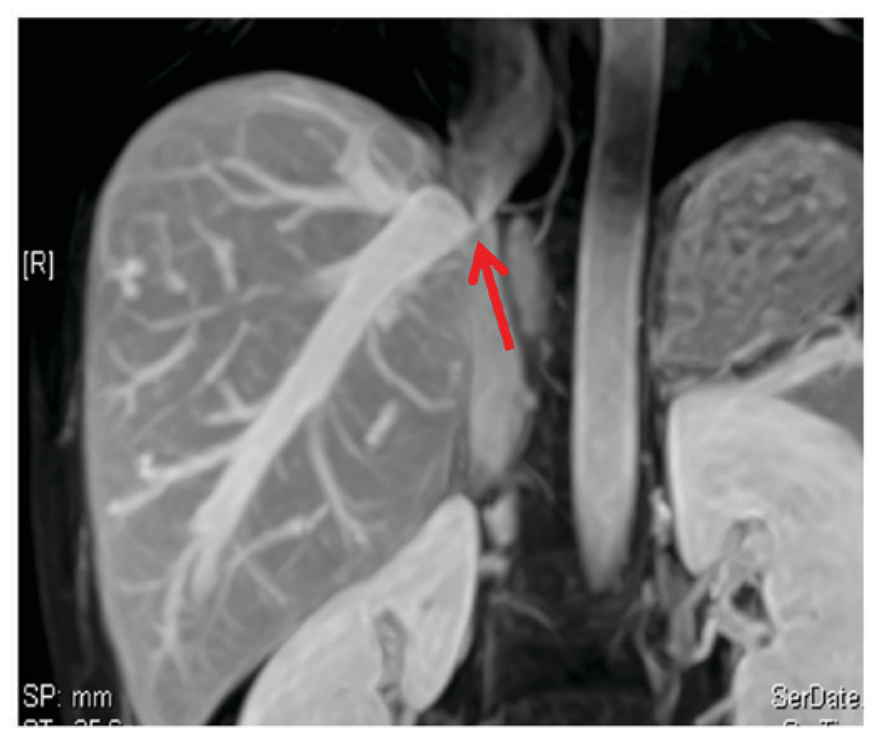

Figure 1. Magnetic resonance image of a patient with Budd-Chiari syndrome due to hepatic vein obstruction revealing membranous obstruction of the right hepatic vein (arrow).

lateral orifice was within the thrombus. Recanalization of the HV was performed after the thrombosis was completely dissolved, or if two consecutive reviews did not indicate any progression of the thrombosis.

TIPS. Through the transjugular route, angiography was performed to identify the major HVs. If the major $\mathrm{HV}$ could not be identified by angiography of the HV, IVC and portal vein, angiography was performed using ultrasound-guided percutaneous liver puncture through an accessory HV. The puncture site of the HV or IVC and portal vein was selected based on the angiography results and TIPS was performed $(9,10)$.

Second-stage treatment. For patients with BCS who did not undergo $\mathrm{HV}$ angioplasty for the first time, a different treatment plan was selected based on the patient's liver function (Child's score) (18). If the patient's Child's score was less than 12 points, medical treatment (including diuresis and liver protection) was given. If medical treatment was effective, $\mathrm{HV}$ angioplasty was performed again following 6 months of medical treatment. If the patient's Child's score exceeded 12 points or medical treatment was not effective, TIPS or liver transplantation was considered.

Post-angioplasty treatment. All patients received subcutaneous low-molecular-weight heparin (5,000 IU; twice daily) for 3 days, and then oral warfarin ( $5 \mathrm{mg}$; daily) for 12 months after treatment. The dose of warfarin was adjusted such that the prothrombin time was maintained at 20-25 sec.

Success criteria for angioplasty. After interventional therapy, if the HVs featured a smooth blood flow and a transmembrane pressure difference of $<4 \mathrm{cmH}_{2} \mathrm{O}$, the procedure was considered successful.

Follow-up. The two centers routinely followed the patients up by telephone and through outpatient services every week for 


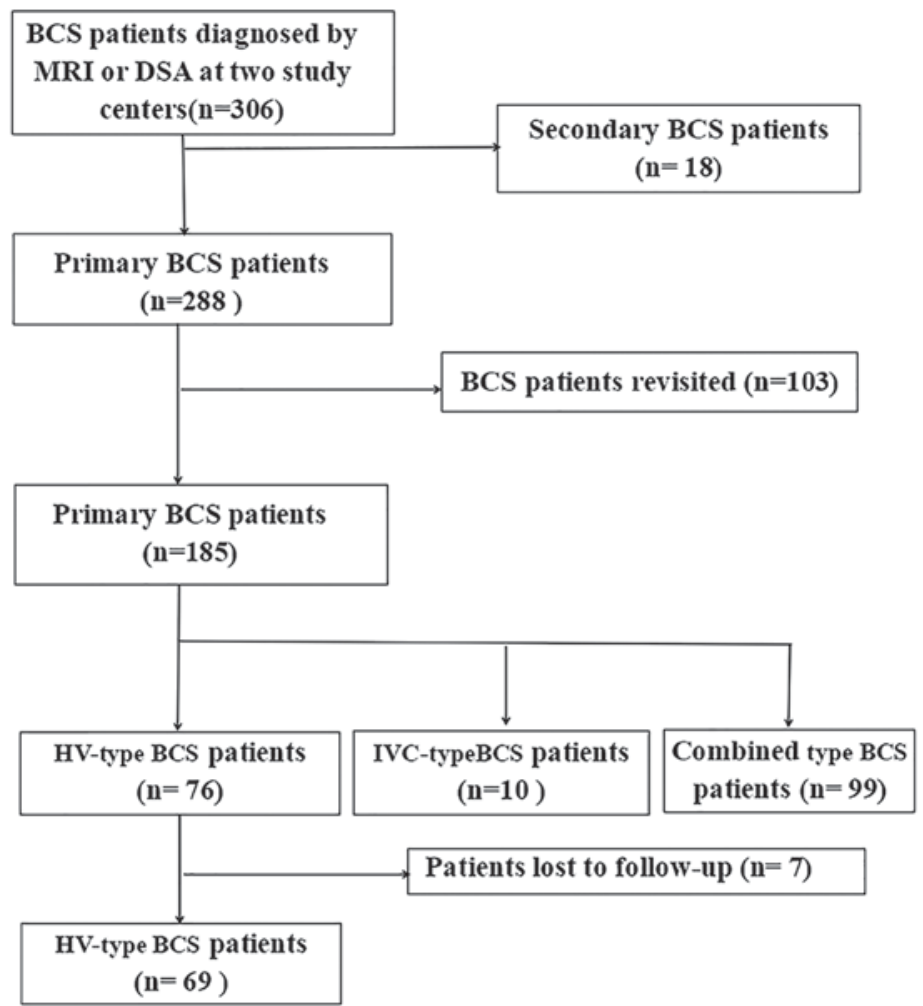

Figure 2. Flowchart of patient selection. BCS, Budd-Chiari syndrome; HV, hepatic vein; MRI, magnetic resonance imaging; DSA, digital subtraction angiography; IVC, inferior vena cava.

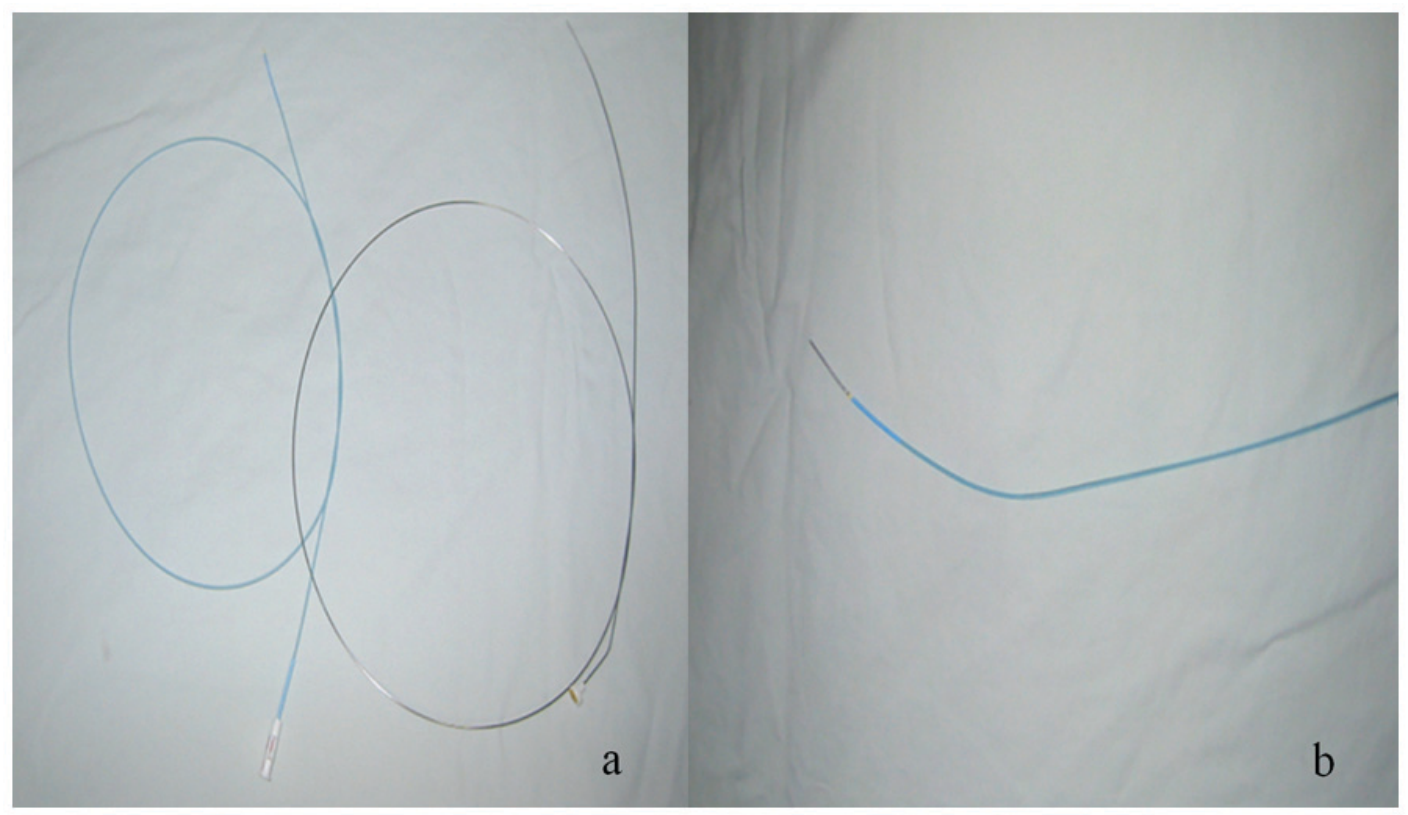

Figure 3. (A) Single-bend catheter and (B) self-made single-bend needle.

the first month, monthly for the next 3 months, every 3 months thereafter. Significant clinical events were recorded, including clinical deterioration, new radiographic signs on the liver and new BCS-associated interventions. Clinical deterioration was defined as re-admission after discharge, occurrence of new symptoms, recurrence of massive ascites, venous dilation over the trunk, leg edema, variceal bleeding or hepatic encephalopathy. The deadline for the follow-up was July 2017, the time-point of death or the time after which the patient was lost to follow-up.

One assigned clinician was responsible for collecting the data for each of the participating patients. The recorded data included socio-demographic features, clinical manifestations, radiology results, interventional treatments and outcomes. Another clinician checked and assessed the data monthly. 


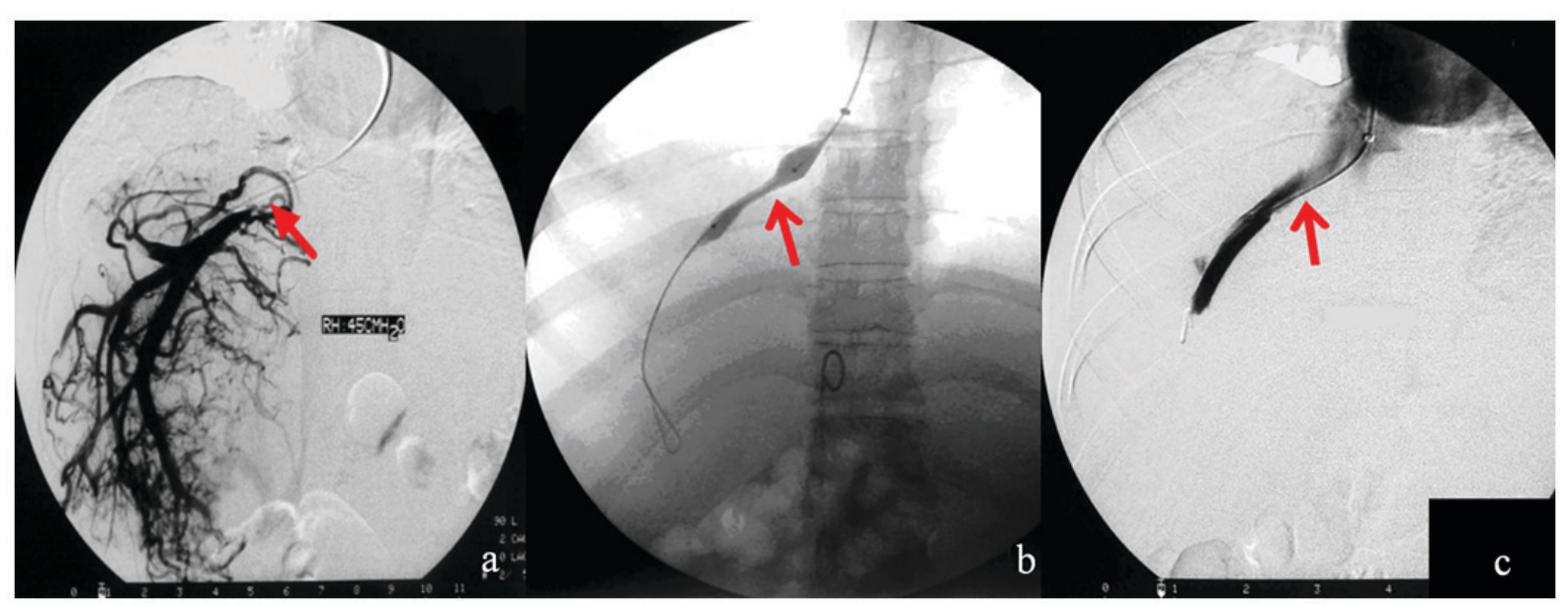

Figure 4. Recanalization of obstructed hepatic vein in patients with Budd-Chiari syndrome via the transjugular approach: (A) Pre-operative angiography of the right hepatic vein indicating occlusion of the ostium (arrow); (B) balloon angioplasty displaying hourglass sign (arrow); (C) post-procedure angiography revealed good blood flow through the hepatic vein after angioplasty (arrow).

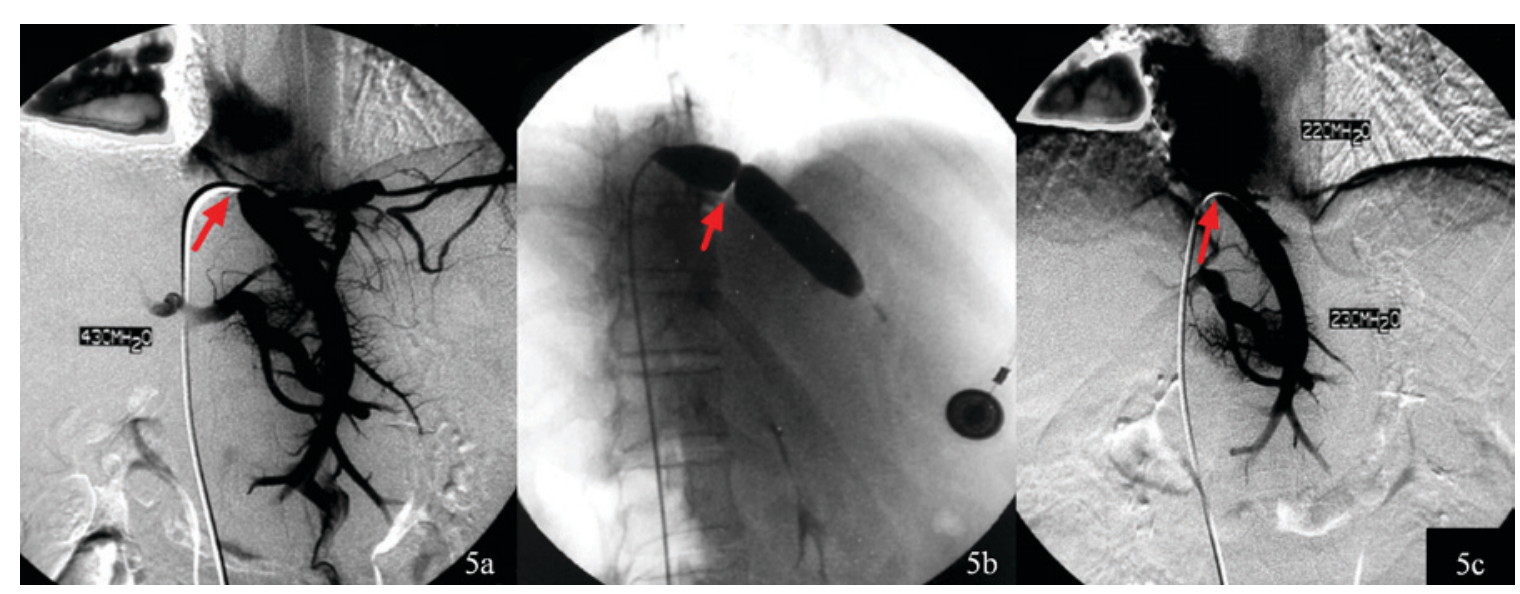

Figure 5. Recanalization of the hepatic vein in patients with hepatic vein obstruction-associated Budd-Chiari syndrome via the femoral vein approach. (A) Pre-operative angiography of the middle hepatic vein showing occlusion of the ostium (arrow); (B) balloon angioplasty showing hourglass sign (arrow); (C) post-angioplasty image showing good blood flow through the middle hepatic vein (arrow).

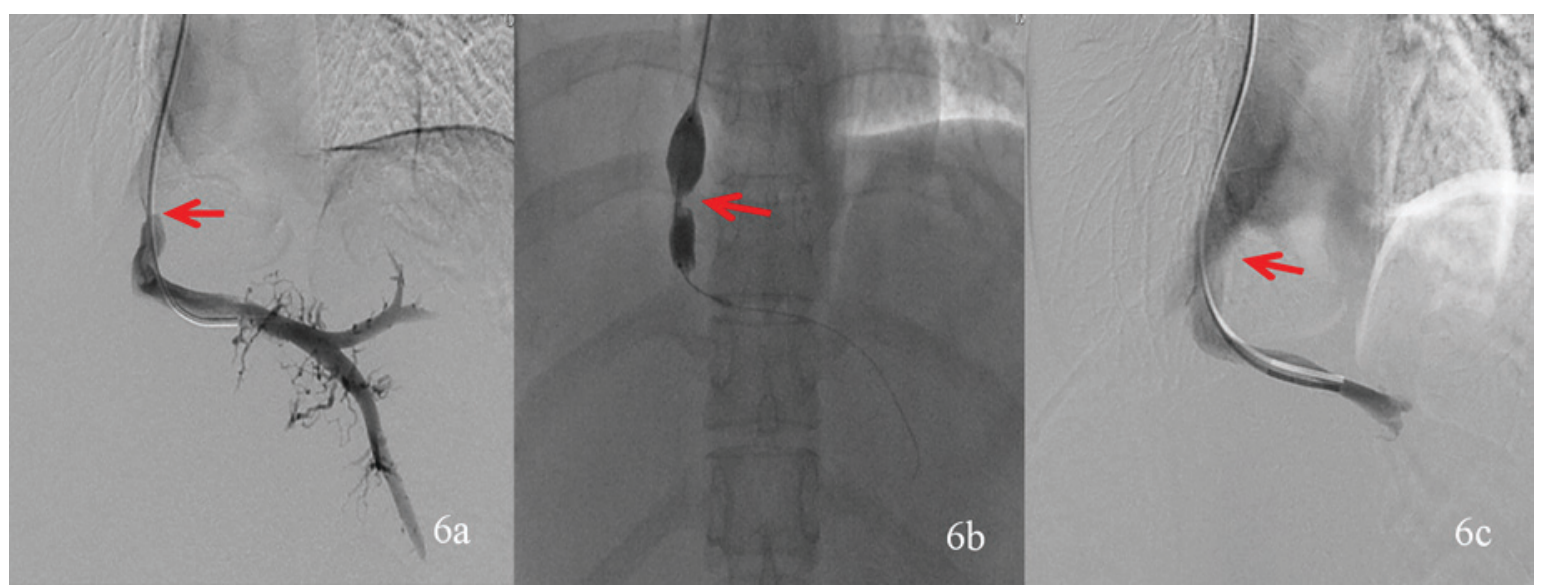

Figure 6. Recanalization of the hepatic vein in patients with hepatic vein obstruction-associated Budd-Chiari syndrome via the transjugular approach: (A) Pre-operative angiography of the left hepatic vein indicating occlusion of the ostium (arrow); (B) balloon angioplasty displaying hourglass sign (arrow); (C) post-procedure angiography revealed good blood flow through the hepatic vein after angioplasty (arrow).

Statistical analysis. SPSS statistical software version 22.0 (IBM Corp., Armonk, NY, USA) was used for the analyses.
Categorical dataare presented as $\mathrm{n}(\%)$ and quantitative data as the mean \pm standard deviation. Quantitative data that 
Table I. Baseline clinical features of patients with Budd-Chiari syndrome $(n=69)$.

\begin{tabular}{lc}
\hline Characteristic & Value \\
\hline Male/female & $43 / 26(62 / 38)$ \\
Age (years) & $43(15-72)$ \\
Smokers $^{\mathrm{a}}$ & $15(22)$ \\
Alcoholics $^{\mathrm{a}}$ & $12(17)$ \\
Duration of symptoms (months) $^{\text {Acute/chronic }}{ }^{\mathrm{b}}$ & $98(0.05-348)$ \\
Abdominal pain & $10 / 59(14 / 86)$ \\
Abdominal distension & $15(22)$ \\
Ascites & $23(33)$ \\
Gastrointestinal bleeding & $35(51)$ \\
Varices of abdominal wall & $16(23)$ \\
Anorexia & $37(54)$ \\
Hepatomegaly & $12(18)$ \\
Splenomegaly & $51(74)$ \\
\end{tabular}

Values are expressed as $\mathrm{n}(\%)$ or median (range). ${ }^{\text {a Smoking, }}$ $>5$ times/day; alcohol intake, $>50 \mathrm{~g} /$ day. ${ }^{\mathrm{b}}$ Acute and chronic defined as symptoms $\leq$ and $>6$ months, respectively.

conformed to a normal distribution (e.g., venous pressure) were analyzed using Student'st-test. Quantitative data that did not conform to a normal distribution were analyzed using the Wilcoxon rank-sum test, and qualitative data (e.g., ascites grade) were analyzed using the $\chi^{2}$ test. Kaplan-Meier curves were drawn to analyze patient survival and the log-rank test was used to analyze differences in survival rates. $\mathrm{P}<0.05$ was considered to indicate a statistically significant difference.

\section{Results}

Patient enrolment. From December 2010 to December 2012, 350 patients with BCS were treated at the two centers (Fig. 2). In accordance with the exclusion criteria, patients with secondary BCS, patients with BCS were diagnosed prior to admission and those with involvement of IVC were excluded. Of the remaining 80 patients with BCS with HVO only, 11 were excluded from the study due to insufficient follow-up. Thus, 69 patients (43 male, 26 female; mean age, 43 years; mean duration of symptoms, 98 months) were included in the analysis. The baseline clinical characteristics and laboratory tests of patients with BCS are presented in Tables I and II).

Success rate of endovascular therapy. Among the 69 patients, primary HV recanalization was successful in 63 cases. Primary therapy failed in 6 patients due to extensive HVO. Of these patients, 2 with severe symptoms received TIPS (post-operatively, the symptoms of one patient resolved and the other one succumbed to liver failure). The remaining 4 patients with mild symptoms received conservative treatment (3 successfully received second-stage recanalization 6 months later). The cumulative technical success rate for $\mathrm{HV}$

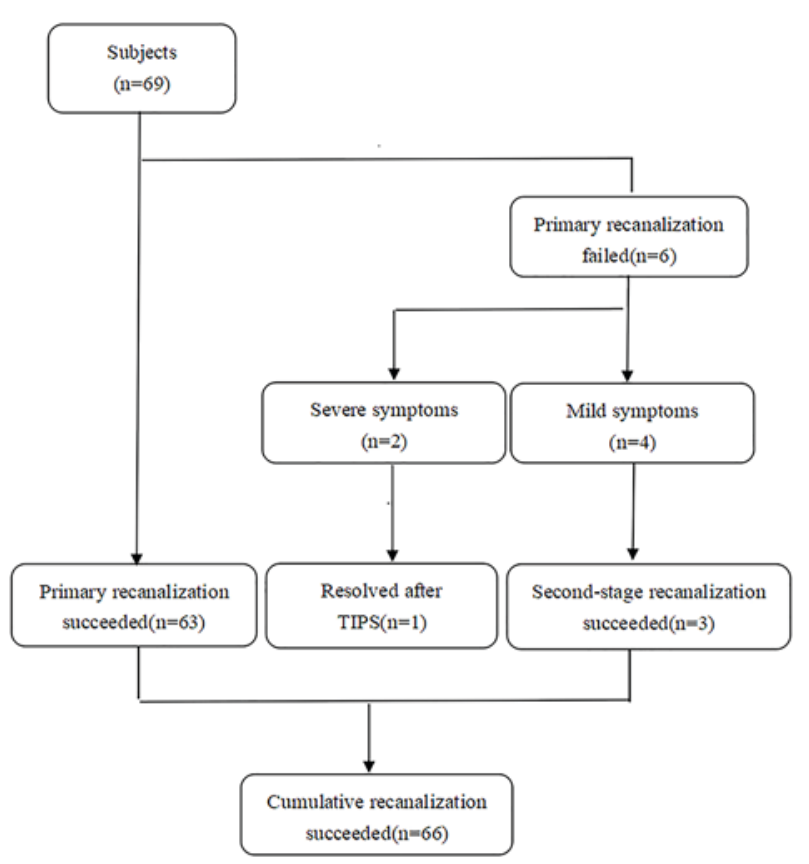

Figure 7. Cumulative technical success rate of hepatic vein recanalization. TIPS, transjugular intrahepatic portosystemic shunt.

recanalization was $95.7 \%$ (66/69; Fig. 7). No serious complications, including pericardial tamponade or ruptured blood vessels, were encountered.

A total of 66 patients successfully underwent angioplasty. Recanalization was performed in 43, 14 and 9 patients via the transjugular, femoral vein and percutaneous transhepatic approach, respectively. In 35 and 13 patients, the target veins for recanalization were 1 or $>2$ major HVs. In 7 patients, the target veins were an accessory $\mathrm{HV}$ and in 11 patients a major $\mathrm{HV}$ and an accessory HV were recanalized.

All of the patients underwent balloon angioplasty. In 41 patients, balloon angioplasty constituted the sole treatment (balloon angioplasty group). In addition, 14, 6 and 5 patients in the combination therapy group respectively received thrombolysis, endovascular stenting and a combination of thrombolysis and endovascular stenting. The baseline characteristics (including sex, age, duration of symptoms, albumin levels, incidence of ascites and cancer antigen-125) of the balloon angioplasty and combination therapy groups are presented in Table III. The differences of incidence of ascites, albumin levels, cancer antigen-125 levels and duration of symptoms between the two groups were statistically significant (Table III).

Clinical efficacy. On admission, 3, 20 and 43 patients presented with mild, moderate and massive ascites, respectively. After endovascular therapy, resolution of ascites was achieved in 53 patients, while 13 patients had a small amount of residual ascites. The difference in the grade of ascites prior to and after therapy was statistically significant $\left(\chi^{2}=122.250, \mathrm{P}=0.001\right)$. The mean HV pressure after $\mathrm{HV}$ recanalization was significantly lower compared with that prior to the procedure $(23 \pm 7$ vs. $47 \pm 9 \mathrm{cmH}_{2} \mathrm{O} ; \mathrm{t}=17.979, \mathrm{P}=0.001$; Table IV). The symptoms of 61 patients were completely relieved after $\mathrm{HV}$ recanalization, while those of 5 patients were partially relieved. 
Table II. Laboratory results of patients with Budd-Chiari syndrome at diagnosis $(n=69)$.

\begin{tabular}{|c|c|c|c|}
\hline Parameters & Median (range) & Normal range & $\begin{array}{c}\text { Patients with abnormal } \\
\text { values, } \mathrm{n}(\%)\end{array}$ \\
\hline Aspartate aminotransferase (U/l) & $36(17-677)$ & $15-40$ & $24(35)^{\mathrm{a}}$ \\
\hline Alanine aminotransferase (U/l) & $34(8-648)$ & $9-51$ & $17(25)^{\mathrm{a}}$ \\
\hline Glutamyl peptide transferase (U/l) & $83(19-536)$ & $3-50$ & $52(75)^{\mathrm{a}}$ \\
\hline Alkaline phosphatase (U/l) & $118(54-351)$ & $45-125$ & $18(26)^{\mathrm{a}}$ \\
\hline Total bilirubin $(\mu \mathrm{mol} / \mathrm{l})$ & $35.6(8.2-114.7)$ & $1.7-21$ & $49(71)^{\mathrm{a}}$ \\
\hline Direct bilirubin $(\mu \mathrm{mol} / \mathrm{l})$ & $14(4.2-72.3)$ & $0-7.3$ & $49(71)^{\mathrm{a}}$ \\
\hline Albumin (g/l) & $35.6(18.5-48.7)$ & $35-51$ & $24(35)^{\mathrm{b}}$ \\
\hline Prothrombin time (sec) & $16.7(12.5-24.3)$ & $11-13$ & $44(64)^{\mathrm{a}}$ \\
\hline White blood cells $\left(\mathrm{x} 10^{9} / \mathrm{l}\right)$ & $4.86(1.07-12.85)$ & $4-10$ & $4(6)^{a}+25(36)^{b}$ \\
\hline Hemoglobin (g/l) & $116(51-169)$ & $120-165$ & $4(6)^{a}+21(30)^{b}$ \\
\hline Platelets $\left(\times 10^{9} / 1\right)$ & $108(18.3-718)$ & $100-300$ & $5(7)^{\mathrm{a}}+34(49)^{\mathrm{b}}$ \\
\hline Cancer antigen-125 (U/1) & $35.1(7.1-1,032.8)$ & $0-35$ & $31(45)^{\mathrm{a}}$ \\
\hline$\alpha$-fetoprotein (ng/ml) & $4.1(0.85-85.3)$ & $0-25$ & $6(9)^{\mathrm{a}}$ \\
\hline Hepatitis B surface antigen $(\mathrm{ng} / \mathrm{ml})(\mathrm{n})^{\mathrm{c}}$ & 6 & $0-0.18$ & $6(9)$ \\
\hline
\end{tabular}

${ }^{\mathrm{a}}$ Above upper limit of the normal range; ${ }^{b}$ below lower limit of the normal range; ${ }^{c}$ positive cases.

Table III. Baseline characteristics of the balloon angioplasty and combination therapy groups.

\begin{tabular}{|c|c|c|c|c|}
\hline Characteristics & Balloon angioplasty $(n=41)$ & Combination therapy $(n=25)$ & $\chi^{2} / Z$ & $\mathrm{P}$-value \\
\hline Males/female (n) & $26 / 15$ & $16 / 9$ & $\chi^{2}=0.002$ & 0.962 \\
\hline Age (years) & $42(16-72)$ & $32(15-63)$ & $Z=-1.199$ & 0.231 \\
\hline Duration of symptoms (months) & $126(56-348)$ & $12(0.05-76)$ & $Z=-2.854$ & 0.004 \\
\hline Ascites (n) & $15(36.6)$ & $20(80.0)$ & $\chi^{2}=11.752$ & 0.001 \\
\hline Aspartate aminotransferase (U/1) & $34(21-589)$ & $46(17-677)$ & $Z=-0.564$ & 0.573 \\
\hline Alanine aminotransferase (U/l) & $32(12-610)$ & $61(8-648)$ & $Z=-0.313$ & 0.754 \\
\hline Glutamyl peptide transferase (U/l) & $74(19-487)$ & $128(21-536)$ & $Z=-0.555$ & 0.579 \\
\hline Total bilirubin $(\mu \mathrm{mol} / \mathrm{l})$ & $35.6(8.2-83.5)$ & $48.7(10.3-114.7)$ & $Z=-0.716$ & 0.474 \\
\hline Albumin (g/l) & $36.8(24.5-48.7)$ & $28.2(18.5-32.6)$ & $Z=-2.263$ & 0.024 \\
\hline Prothrombin time (sec) & $15.7(12.5-23.6)$ & $17.8(12.8-24.3)$ & $Z=-1.485$ & 0.138 \\
\hline Cancer antigen-125 (U/1) & $29.1(7.1-367.5)$ & $421.5(17.5-1,032.8)$ & $Z=-2.711$ & 0.007 \\
\hline
\end{tabular}

Values are expressed as $\mathrm{n}(\%)$ or median (range).

Survival rate. The median follow-up period was 75 months (range, 60-84 months). At 12, 36 and 60 months, the cumulative survival rate of patients subjected to endovascular interventional therapy was $98.5,98.5$ and $93.9 \%$, respectively, while that in the balloon angioplasty group was 97.6, 97.6 and $97.6 \%$, respectively, and that in the combination therapy group was $96.0,96.0$ and $88.0 \%$, respectively. No significant differences in all cumulative survival rates between the balloon angioplasty group and combination therapy group were identified $\left(\chi^{2}=2.387 ; \mathrm{P}=0.122\right.$; Fig. 8$)$.

\section{Discussion}

In theory, angioplasty of an obstructed $\mathrm{HV}$ may reduce congestion of the liver, decrease pressure of the HV and the portal vein, and restore the patient's liver function, thereby making it an ideal treatment for BCS caused by $\operatorname{HVO}(7,8)$. However, endovascular therapy remains a challenging procedure in these patients due to the anatomical characteristics of the HV $(13,19)$ and the difficulty in restoring HV flow. However, in the present study, the technical success rate of the operation in the Chinese BCS patients with HVO was high $(95.7 \%)$ and the post-operative asymptomatic survival rate $(80.3 \%$ at 5 years) was also high after angioplasty of the obstructed HVs.

In each patient, the first task was to determine which approach to use for recanalization of the obstructed HV. At present, the 3 most commonly used therapeutic approaches are the transjugular, transfemoral and percutaneous transhepatic approach $(4,7)$. In the majority of patients with BCS due to 
Table IV. Pre- and post-operative clinical efficacy according to severity of ascites and HV pressure.

\begin{tabular}{|c|c|c|c|c|}
\hline Parameters & Pre-operative $\mathrm{n}(\%)$ & Post-operative n (\%) & $\chi^{2} / t$-value & P-value \\
\hline Ascites (n) & & & $\chi^{2}=122.250$ & 0.001 \\
\hline None & $0(0)$ & $53(80.3)$ & & \\
\hline Mild & $3(4.5)$ & $13(19.7)$ & & \\
\hline Moderate & $20(30.3)$ & $0(0)$ & & \\
\hline Massive & $43(65.2)$ & $0(0)$ & & \\
\hline $\mathrm{HV}$ pressure $\left(\mathrm{cmH}_{2} \mathrm{O}\right)$ & $47 \pm 9$ & $23 \pm 7$ & $\mathrm{t}=17.979$ & 0.001 \\
\hline
\end{tabular}

Values are expressed as $\mathrm{n}$ or the mean \pm standard deviation. HV, hepatic vein.
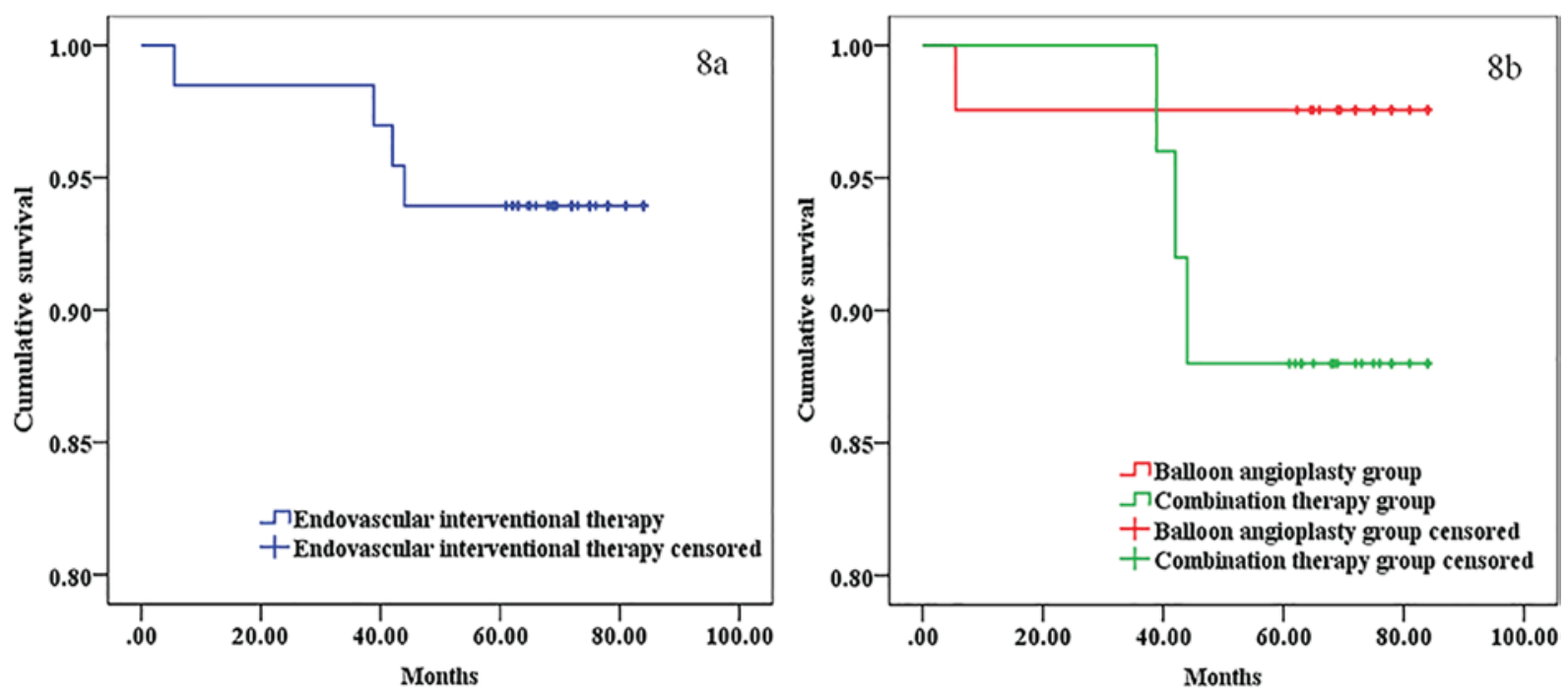

Figure 8. Cumulative survival associated with the treatments.

$\mathrm{HVO}$, the obstruction is in the proximal $\mathrm{HV}$, resulting from stenosis of the ostium or membranous occlusion $(4,14,20)$. These patients are ideally treated by recanalization via the jugular or femoral vein.

The transjugular approach has a high success rate, as the angle between the HV and the proximal IVC is usually relatively large, and the guide wire may easily access the HV via the jugular vein $(4,17,21)$. If recanalization of the $\mathrm{HV}$ via the transjugular fails, the femoral vein may be used, but this is technically more difficult. In certain patients with an accessory $\mathrm{HV}$ that intersects the IVC at an obtuse angle, the transfemoral approach may be more appropriate $(4,11,19,21)$. The percutaneous transhepatic approach may be used in patients with mild ascites, if the jugular and femoral approaches have failed $(4,13)$. For patients with ascites whose extent is more than mild, diuretics and other conservative treatments should be offered initially, and the percutaneous transhepatic approach could be employed once the ascites has significantly reduced.

The second task is to select the appropriate target HV for recanalization. Ideally, the obstructed vein should be the first choice. The right HV drains a large area of the liver, has a smaller angle with the IVC and is the preferred vein for interventional therapy $(7,13,19-21)$. If all 3 major HVs are obstructed, it may be attempted to reopen the right HV first. If the diameter and drainage range of the HVs selected for recanalization are sufficiently large, reperfusion may be effective for relieving the portal hypertension $(4,17,21)$. However, if the diameter of the $\mathrm{HV}$ is small, multiple HVs may be considered for recanalization during the same procedure $(4,7,11,13,18,21)$. If all of the HV segments are occluded, leading to failure of the interventional therapy, recanalization of the accessory $\mathrm{HV}$ may be attempted to relieve portal hypertension $(13,19)$.

The final task was to determine what type of angioplasty was suitable for recanalization of the obstructed $\mathrm{HV}$. In the present study, it was observed that the symptoms completely disappeared if the transmembrane pressure difference after percutaneous transluminal angioplasty was $4 \mathrm{cmH}_{2} \mathrm{O}$. Therefore, it is indicated that the transmembrane pressure difference is a reliable predictor of successful treatment, although specific indicators require to be verified (7). The choice of treatment depends on the location and extent of the obstruction, as well as the characteristics of the lesion $(3,4,8,13,17,21,22)$. For thrombus-free obstruction, balloon angioplasty has been recommended, with the diameter of the selected balloon based on the diameter of the vessel being treated, typically $12-20 \mathrm{~mm}$ (23). If the transmembrane pressure difference is $<4 \mathrm{cmH}_{2} \mathrm{O}$, the treatment is considered 
successful. If the transmembrane pressure difference remains $>4 \mathrm{cmH}_{2} \mathrm{O}$, an endovascular stent is placed. For the thromboembolic $\mathrm{HV}$, thrombolytic therapy has been recommended, followed by balloon angioplasty and endovascular stent $(8,17)$.

For patients with treatment failure and mild clinical symptoms, conservative medical treatment prior to the second stage of medullary treatment, performed after the formation of collateral circulation in the compensated liver, has been suggested $(3,6,8)$. In the present study, 3 patients were successfully subjected to second-stage treatment. In patients with a severe condition, TIPS was required to relieve portal hypertension and increase the chance of survival $(8-10,24)$. For certain cases, liver transplantation has also been recommended $(5,8)$.

In the present study, no significant differences in symptom-free survival rates were identified between the balloon angioplasty and combination treatment groups. This may be due to the small sample size. Furthermore, in the present cohort was not randomized regarding the interventional treatment, but it was selected based on the pathological features of each patient, which may have introduced selection bias. Therefore, even if the post-operative asymptomatic survival rate of patients receiving a specific treatment was higher than that of patients receiving other treatments, this does not confirm the superiority of the treatment.

Re-obstruction of the vein is a post-operative complication and the underlying cause of recurrence of BCS symptoms (4). According to various studies, HV stenting may reduce the incidence of HV re-obstruction $(3,8,16,25)$. However, stents are permanent devices that, increase the difficulty of a subsequent interventional procedure in the case of a recurrent stenosis, and should therefore be used with caution. A large multicenter study indicated that the patency rates of covered and bare stents in patients with BCS were comparable (6). In a patient with BCS due to HVO, a covered stent affected the formation of a compensatory collateral circulation and further aggravated hepatic ischemia (12). Therefore, the use of a covered stent for recanalization of BCS caused by HVO may not be recommended.

BCS associated with HVO may be classified as stenosis, membranous or segmental occlusion, thrombosis or wide occlusion $(1,3,4)$. For stenosis or membranous/segmental occlusion, angioplasty has been effective and has a high post-operative asymptomatic survival rate $(4,13,17)$. In the present study, the majority of patients recovered after endovascular interventional therapy, and the cumulative survival rate at 60 months post-operatively was $93.9 \%$. The cumulative survival rate at 60 months in the balloon angioplasty group (97.6\%) was higher than that in the combination therapy group (88.0\%). This is probably due to the fact that most of the patients who received combination therapy had more severe pre-operative symptoms such as ascites and complicated baseline conditions (lower serum albumin), which were associated with a relatively poorer prognosis. However, since the sample size was relatively small, the difference between the 2 groups was not statistically significant, and further study with a larger population is required.

For patients with extensive obstruction, it is more difficult to recanalize via angioplasty, with high rates of failure and low rates of symptom-free survival. These patients are better treated by TIPS, which directly reduces portal pressure, relieves sinus pressure and has a higher efficacy $(3,9,10)$.
In the present study, the post-operative symptom-free survival rates at 12,36 and 60 months were 98.5, 98.5 and $92.4 \%$, respectively. These are slightly higher than the survival rate (97.7, 92.2, and 90.0\%) reported by Cui et al (4). In the present study, as most patients with BCS had HV stenosis or membranous or segmental occlusion, the outcomes of recanalization were good. However, only $29-41 \%$ of Western patients with BCS have membranous or segmental occlusion of the HV $(4,26,27)$. Therefore, percutaneous recanalization is not applicable to most Western patients with BCS $(4,26,27)$, and TIPS is the recommended treatment $(3,9,10)$. The putative 6 - to- 120 -month survival rates of Western patients with $\mathrm{HV}$-associated $\mathrm{BCS}$ after TIPS were 72-97\% $(10,26)$, which is slightly lower than the $12-$, 36- and 60-month survival rate determined in the present study. This may be due to the condition being more complicated in most Western patients with BCS receiving TIPS, and therefore, their survival rate is relatively low. These differences between Eastern and Western patients with HV-associated BCS may explain for the differences in the reported rates of recanalization. However, regardless of these differences, in the present study, the mid-term asymptomatic survival rate was high. This indicates that recanalization was effective for the treatment of $\mathrm{HV}$-associated BCS in Chinese patients.

The present study is limited by its retrospective nature and small sample size. In addition, the patients were not randomized regarding the mode of endovascular treatment, but the treatment was selected based upon the pathological features of the patients, which may have introduced bias. Hence, infuture, large sample prospective studies are required to validate the results of the present study.

In conclusion, in the Chinese cohort of the present study, the majority of patients with BCS had segmental or membranous HVO. In the current study, the endovascular interventional treatment of BCS patients with HVO that was applied based on the characteristics of $\mathrm{HV}$ vascular lesions. These patients experienced high symptom-free survival rates and low recurrence rates after endovascular therapy. Larger prospective studies are required to confirm the efficacy of endovascular therapy for BCS caused by HVO.

\section{Acknowledgements}

Not applicable.

\section{Funding}

The present study was supported by Natural Science Foundation of Anhui Province (grant no. 1708085QH218).

\section{Availability of data and materials}

All data generated or analyzed during the present study are included in this published article.

\section{Authors' contributions}

DC, NZ and CL conceived and designed the study. HX, WF and $C L$ were responsible for the collection and analysis of the patient data. DC, NZ and CL interpreted the data and drafted the manuscript. WL contributed to the conception and design 
of this study and revised the manuscript critically for important intellectual content. All authors read and approved the final manuscript.

\section{Ethical approval and consent to participate}

All procedures were performed in accordance with the ethical standards of the responsible committee on human experimentation (institutional and national) and with the Helsinki Declaration of 1975, as revised in 2008 (5). Informed consent was obtained from all patients for being included in the study.

\section{Patient consent for publication}

Not applicable.

\section{Competing interests}

The authors declare that they have no competing interests.

\section{References}

1. Martens P and Nevens F: Budd-Chiari syndrome. United European Gastroenterol J 3: 489-500, 2015.

2. Shin N, Kim YH, Xu H, Shi HB, Zhang QQ, Colon Pons JP, Kim D, Xu Y, Wu FY, Han S, et al: Redefining Budd-Chiari syndrome: A systematic review. World J Hepatol 8: 691-702, 2016.

3. Goel RM, Johnston EL, Patel KV and Wong T: Budd-Chiari syndrome: Investigation, treatment and outcomes. Postgrad Med J 91: 692-697, 2015.

4. Cui YF, Fu YF, Li DC and Xu H: Percutaneous recanalization for HV-type Budd-Chiari syndrome: Long-term patency and survival. Hepatol Int 10: 363-369, 2016.

5. Akamatsu N, Sugawara Y and Kokudo N: Budd-Chiari syndrome and liver transplantation. Intractable Rare Dis Res 4: 24-32, 2015.

6. Darwish Murad S, Plessier A, Hernandez-Guerra M, Fabris F, Eapen CE, Bahr MJ, Trebicka J, Morard I, Lasser L, Heller J, et al Etiology, management, and outcome of the Budd-Chiari syndrome. Ann Intern Med 151: 167-175, 2009.

7. Sang HF and Li XQ: Endovascular treatment of Budd-Chiari syndrome with HV obstruction in China. J Laparoendosc Adv Surg Tech A 24: 846-851, 2014.

8. Seijo S, Plessier A, Hoekstra J, Dell'era A, Mandair D, Rifai K, Trebicka J, Morard I, Lasser L, Abraldes JG, et al: Good long-term outcome of Budd-Chiari syndrome with a step-wise management. Hepatology 57: 1962-1968, 2013.

9. Fitsiori K, Tsitskari M, Kelekis A, Filippiadis D, Triantafyllou K and Brountzos E: Transjugular intrahepatic portosystemic shunt for the treatment of Budd-Chiari syndrome patients: Results from a single center. Cardiovasc Intervent Radiol 37: 691-697, 2014.

10. Tripathi D, Macnicholas R, Kothari C, Sunderraj L, Al-Hilou H, Rangarajan B, Chen F, Mangat K, Elias E and Olliff S: Good clinical outcomes following transjugular intrahepatic portosystemic stent-shunts in Budd-Chiari syndrome. Aliment Pharmacol Ther 39: 864-872, 2014.

11. Cheng D, Xu H, Lu ZJ, Hua R, Qiu H, Du H, Xu X and Zhang J: Clinical features and etiology of Budd-Chiari syndrome in Chinese patients: A single-center study. J Gastroenterol Hepatol 28: 1061-1067, 2013.
12. Qi XS, Ren WR, Fan DM and Han GH: Selection of treatment modalities for Budd-Chiari syndrome in China: A preliminary survey of published literature. World J Gastroenterol 20: 10628-10636, 2014.

13. Cui YF, Fu YF, Wei N, Zhu HC and Xu H: Retrograde puncture assisted HV recanalization in treating Budd-Chiari syndrome with segmental obstruction of hepatic venous. Radiol Med 120: 1184-1189, 2015

14. Shalimar Kumar A, Kedia S, Sharma H, Gamanagatti SR, Gulati GS, Nayak B, Thakur B and Acharya SK: Hepatic venous outflow tract obstruction: Treatment outcomes and development of a new prognostic score. Aliment Pharmacol Ther 43: 1154-1167, 2016.

15. Meng X, Lv Y, Zhang B, He C, Guo W, Luo B, Yin Z, Fan D and Han G: Endovascular management of Budd-Chiari syndrome with IVC thrombosis: A 14-year single-center retrospective report of 55 patients. J Vasc Interv Radiol 27: 1592-1603, 2016.

16. Huang Q, Shen B, Zhang Q, Xu H, Zu M, Gu Y, Wei N, Cui Y and Huang R: Comparison of long-term outcomes of endovascular management for membranous and segmental IVC obstruction in patients with primary Budd-Chiari syndrome. Circ Cardiovase Interv 9: e003104, 2016.

17. Zhang Q, Xu H, Zu M, Gu Y, Wei N, Wang W, Gao Z and Shen B: Catheter-directed thrombolytic therapy combined with angioplasty for hepatic venous obstruction in Budd-Chiari syndrome complicated by thrombosis. Exp Ther Med 6: 1015-1021, 2013.

18. Siriwardana RC, Niriella MA, Dassanayake AS, Liyanage C, Gunathilaka B, Jayathunge S and de Silva HJ: Clinical characteristics and outcome of hepatocellular carcinoma in alcohol related and cryptogenic cirrhosis: A prospective study. Hepatobiliary Pancreat Dis Int 14: 401-405, 2015.

19. Tang W, Zhang XM, Yang L, Mitchell DG, Zeng NL and Zhai ZH: Hepatic caudate vein in Budd-Chiari syndrome: Depiction byusing magnetic resonance imaging. Eur J Radiol 77: 143-148, 2011.

20. Mukund A and Gamanagatti S: Imaging and interventions in Budd-Chiari syndrome. World J Radiol 3: 169-177, 2011.

21. Gao Y, Chen S and Yu C: Applicability of different endovascular methods for treatment of refractory Budd-Chiari syndrome. Cell Biochem Biophys 61: 453-460, 2011.

22. Fan X, Liu K, Che Y, Wang S, Wu X, Cao J and Li J: Good clinical outcomes in Budd-Chiari syndrome with hepatic venous occlusion. Dig Dis Sci 61: 3054-3060, 2016.

23. Hanaoka J, Shimada M, Uchiyama H, Ikegami T, Imura S, Morine $\mathrm{Y}$ and Kanemura $\mathrm{H}$ : A simple formula to calculate the liver drainage volume of the accessory right $\mathrm{HV}$ using its diameter alone. Surgery 146: 264-268, 2009.

24. Miraglia R, Maruzzelli L and Luca A: Recanalization of occlusive transjugular intrahepatic portosystemic shunts inaccessible to the standard transvenous approach. Diagn Interv Radiol 19: $61-65,2013$

25. Gupta AC, Wang W, Shah C, Sands MJ, Bullen J, Remer EM, Bayona PM, Carey W and Kapoor B: Added value of covered stents in transjugular intrahepatic portosystemic shunt: A large single-center experience. Cardiovasc Intervent Radiol 40: 1723-1731, 2017.

26. Eapen CE, Velissaris D, Heydtmann M, Gunson B, Olliff S and Elias E: Favourable medium term outcome following hepatic vein recanalisation and/or transjugular intrahepatic portosystemic shunt for Budd Chiari syndrome. Gut 55: 878-884, 2006.

27. Valla D, Hadengue A, el Younsi M, Azar N, Zeitoun G, Boudet MJ, Molas G, Belghiti J, Erlinger S, Hay JM and Benhamou JP: Hepatic venous outflow block caused by short-length hepatic vein stenoses. Hepatology 25: 814-819, 1997.

(i) $($ This work is licensed under a Creative Commons Attribution-NonCommercial-NoDerivatives 4.0 International (CC BY-NC-ND 4.0) License. 